\title{
Cuatro años del Gobierno de Mauricio Funes
}

El Salvador es sacudido por marejadas de un ciclo político electoral que comenzó con las elecciones de 2012, continuará con la elección presidencial de 2014 y terminará con los comicios municipales y legislativos de 2015. Tres elecciones en tres años que costarán cerca de cien millones de dólares ${ }^{1}$. Una carga demasiado pesada para un país con sus finanzas maltrechas, demasiado tediosa para la población y un factor que no abona al abordaje serio y fundamentado de los grandes problemas de la sociedad. Diversas voces, entre ellas la del magistrado presidente del Tribunal Supremo Electoral, han hecho pública la conveniencia de revisar el itinerario electoral. No había que echar en saco roto esta necesidad. Por supuesto, la implementación del voto en el exterior no es negociable, porque es una obligación que el Gobierno le adeuda a quienes mantienen a flote la economía de un país que les negó la oportunidad de sobrevivir en su propia tierra. El asunto está en revisar los calendarios electorales, a fin de hacer coincidir las elecciones de manera que haya más tiempo de respiro para la población y para el país.

En este ambiente de cuasi permanentes campañas, arribó a sus cuatro años de gestión el Gobierno presidido por Mauricio Funes, un ambiente que tiñe las valoraciones de su gestión con los colores que convienen a los fines electorales de la oposición, del partido gobernante y del mismo Gobierno. Arena ha presentado sus particulares visiones de la gestión del Gobierno, con la anuencia de los medios de comunicación de mayor cobertura que, para ello, convocan a "analistas" reconocidos por sus posicionamientos críticos contra el Gobierno. Destaca, en este apartado, la valoración muy publicitada de la gran empresa privada para quien este Gobierno representa un panorama sombrío, de constantes fracasos y pésimas decisiones. En cuanto al Gobierno, emprendió una campaña caracterizada por la grandilocuencia que sobredimensiona los logros de lo que consideran un gobierno de cambios positivos para el país, enfatizando la gestión de la figura del presidente. Y por su parte el FMLN, por primera vez en cuatro años, capitaliza la sobresaliente calificación que la población da a la administración Funes y asume como propia la gestión gubernamental, con la complicidad silenciosa del presidente, que al inicio de su gestión se empeñó en separar Gobierno y partido.

1. De acuerdo al TSE, las elecciones de 2012 tuvieron un costo de 32.9 millones de dólares; el presupuesto aprobado por el Ministerio de Hacienda para las elecciones presidenciales de 2014 ronda la misma cantidad (aunque el TSE solicitó 44.2 millones por la posibilidad de que sean necesarios dos eventos electorales, más la implementación del voto residencial a nivel nacional y del voto en el exterior). Estimamos que las elecciones legislativas y municipales de 2015 costarán una cantidad similar. 
Ya estamos acostumbrados a que la violencia sea percibida como uno de los dos grandes problemas del país. Sin embargo, el hecho de que, en el contexto de la significativa reducción de homicidios por la tregua entre las pandillas, la percepción de la gente siga poniendo la inseguridad en el primer lugar de sus preocupaciones es un fuerte llamado de atención al Gobierno
En este contexto, hacer una valoración que pretenda ser más objetiva exige tomar distancia de las apreciaciones subjetivas e ideologizadas. Una de las posibles claves para no morir en el intento es poner atención a lo que la población piensa, opina, percibe. Las encuestas son un mecanismo que, con métodos estadísticos, intentan obtener, a partir de una muestra de población seleccionada científicamente, la percepción de todo el conglomerado. Al menos, esta es la filosofía del trabajo del Instituto Universitario de Opinión Pública (IUDOP), cuyos resultados de su última encuesta de mayo de 2013 serán nuestra guía para dar nuestro punto de vista sobre los cuatro años de gestión del primer Gobierno de izquierda del país, centrándonos en lo que, de acuerdo a la opinión de la población, son los dos principales problemas: la violencia y la situación económica.

\section{Las mismas piedras: violencia y economía}

Si en algo hay absoluta coincidencia en todas las encuestas y en todos los análisis es en catalogar como los dos principales problemas del país y, por tanto, como las dos grandes deudas del presente Gobierno, la situación de violencia-inseguridad y la crisis económica.

Ya estamos acostumbrados a que la violencia sea percibida como uno de los dos grandes problemas del país. Sin embargo, el hecho de que, en el contexto de la significativa reducción de homicidios por la tregua entre las pandillas, la percepción de la gente siga poniendo la inseguridad en el primer lugar de sus preocupaciones es un fuerte llamado de atención al Gobierno. De acuerdo a la encuesta del IUDOP, $40.7 \%$ de la gente opina que la delincuencia es el principal problema que enfrenta el país, porcentaje superior al $22.4 \%$ que opina que es la economía. Al agrupar los problemas que tienen que ver con la violencia (delincuencia, violencia y maras), resulta que más de la mitad de la población (53.4 \%) considera que, en este ámbito, está el principal problema del país, por encima también del porcentaje resultante de agrupar las respuestas de carácter económico (economía, desempleo y pobreza) que es del $43 \%$. La difícil situación que vive la sociedad salvadoreña hace que 38 de cada 100 personas tenga deseos de migrar, porcentaje también mayor a los obtenidos en ejercicios anteriores.

La lógica de la gente es coherente con esta percepción a lo largo de toda la encuesta del IUDOP. Como principal tarea para el quinto año de gestión se le encomienda, a la Administración Funes, combatir la delincuencia (37.5 \%) y resolver los problemas en el orden económico (mejorar la economía, controlar el costo de la canasta básica, crear fuentes de empleo, combatir la pobreza), que suman, entre todos, el $41.9 \%$ de las opiniones. Como resumen de las percepciones anteriores, casi la mitad de la población (45.8 \%) estima 
que la situación económica ha empeorado y el $41.3 \%$ piensa que la delincuencia también ha aumentado durante el presente Gobierno.

\section{Un Gobierno bien evaluado... ¿por qué?}

Después de cuatro años de gobierno, la administración del presidente Funes ha sido muy bien calificada por la población en las diversas encuestas de opinión, siendo esta la otra gran coincidencia de las encuestas que no se da en las opiniones de los analistas.

En la encuesta del IUDOP, el presidente y su Gobierno fueron calificados con 6.87, nota muy cercana a la que se le adjudicó en sus dos primeros años, y muy por encima de las notas de sus predecesores a estas alturas de la gestión. Más de la mitad de la población consideró que el Gobierno ha representado un cambio positivo para el país (60.2\%), y el $71.3 \%$ de la gente considera que la gestión de Funes ha sido buena o muy buena en el último año. En definitiva, el actual Gobierno se erige como el mejor evaluado por la población después de la guerra y, de acuerdo a comparaciones con otros países, uno de los mejor evaluados a nivel latinoamericano.

Tomando en cuenta esta positiva evaluación de la gestión gubernamental y la constatación de que la crisis económica y la situación de violencia e inseguridad son las dos grandes preocupaciones del pueblo salvadoreño, incluso en niveles más alarmantes que en años anteriores, ¿̇cómo es posible que el Gobierno y su presidente salgan tan bien evaluados? Más concretamente, ¿qué hace que, a pesar de esta difícil situación, el Gobierno salga bien ponderado por la población?

Sin lugar a dudas, la destacada calificación que la población le da al presidente y su Gobierno a estas alturas de su gestión se debe, en gran medida, a los programas sociales que ha implementado, algunos de los cuales tienen que ver con ayuda económica a personas y a familias pobres ${ }^{2}$. En la encuesta del IUDOP, a la pregunta de cuál es el principal logro de la gestión del Gobierno, el mayor porcentaje de opiniones (36.7 \%) se concentró en la entrega de los paquetes escolares (útiles, uniformes, zapatos), y además la gente consideró que esto representa el cambio más positivo para el país (39.9 \%). El programa de Ciudad Mujer, una iniciativa de la Secretaría de Inclusión Social, presidida por la primera dama, fue considerado como la promesa mejor cumplida por el $83.9 \%$ de las personas encuestadas. También, otras promesas hechas por el presidente durante su campaña fueron evaluadas como cumplidas, por una buena parte de los encuestados: dotación de viviendas (48.6\%), mejora en la salud pública (57.9\%), mejora en el sistema educativo (75.2 \%) y el acceso a la educación (66.2 \%).

2. El Gobierno de Mauricio Funes implementa ocho programas sociales: Paquetes Escolares, Alimentación Escolar, Vaso de Leche, Paquetes Agrícolas, Pensión Básica para Adultos Mayores, Programa de Atención Temporal al Ingreso (PATI), Comunidades Solidarias Rurales y Urbanas, y Ciudad Mujer. 
En cuanto a la evaluación de las carteras de Estado, las secretarías encargadas de los programas sociales han sido muy bien evaluadas por la población: Educación (7.79), Agricultura y Ganadería (7.29), Inclusión Social (7.13). Hay que mencionar también la buena nota dada por la población a la Secretaría de Obras Públicas (7.49, la segunda más alta) y de Turismo (7.48, la tercera), las cuales seguramente han abonado a la calificación global del Gobierno.

De acuerdo a reportes del Gobierno, los ocho programas sociales que implementa, en conjunto, estarían beneficiando directamente a más de millón y medio de personas de escasos recursos económicos.

Al cruzar las variables en la encuesta del IUDOP, los resultados revelan que, precisamente, son los sectores beneficiados por estos programas los que mejor califican al Gobierno. Las mejores notas a su desempeño provienen de la zona rural (7.17), los sectores obreros (6.84), las personas de menor nivel educativo (7.36), las personas de 56 años o más (6.99), las mujeres (6.97), las personas de la zona oriental (7.35) y paracentral (7.04) y los que declararon tener como partido de preferencia al FMLN (7.95). Es decir, son los sectores precisamente beneficiados con los programas sociales. Por contrapartida, las peores notas adjudicadas a la gestión del Gobierno provienen de la zona metropolitana (6.62), de los sectores con mayores ingresos (6.31) $y$ de medianos ingresos (6.34), los de la zona marginal (6.40), los que manifestaron no tener partido de preferencia (6.48) y los que dicen simpatizar con Arena (5.79).

Una percepción de la población que viene a corroborar lo dicho arriba es la respuesta que dio a la pregunta "¿A qué grupo ha beneficiado más el actual Gobierno?". El 46 \% de las personas entrevistadas contestó: "A los pobres". La respuesta que más porcentaje obtuvo después de esta (9.3\%) fue: "A todos por igual".

\section{¿Cuál es el mensaje de la población?}

¿Hay contradicción si la misma persona opina que la economía y la situación de inseguridad han empeorado durante esta gestión y, simultáneamente, cree que tiene un buen Gobierno?

Varios factores pueden explicar esta aparente contradicción. En primer lugar, como ya se señaló, los sectores más pobres que son beneficiarios de los programas sociales son los que mejor opinión tienen sobre la gestión del Gobierno. Y aunque estos programas sociales son descalificados con mucha facilidad por la oposición y por varios analistas que los consideran populistas, representan una ayuda fundamental para las familias de más escasos recursos económicos. Probablemente, los sectores medios urbanos y los más ricos de este país, al no conocer de cerca la dureza de la realidad que viven los más pobres, no tienen idea de lo importante que son para ellos esas ayudas que con ligereza se descalifican como asistencialistas y que algunos consideran un despilfarro. Es cierto que esos programas no solucionan los 
problemas estructurales de los sectores de bajos ingresos del país, pero durante veinte años no tuvieron ni siquiera eso. Los programas sociales son una gran ayuda, insuficiente si se quiere, pero tienen como destinatarios a sectores que han estado en el olvido desde siempre.

En segundo lugar, de acuerdo a la percepción de la gente, el Gobierno no es el responsable de todos los males que vive la sociedad salvadoreña. Al preguntar sobre los responsables de la crítica situación del país, el 38 \% respondió que es la crisis mundial y el $24.4 \%$ cree que es responsabilidad de los Gobiernos anteriores. Una frase repetida por personas que apoyan la gestión del actual Gobierno - "hace lo que se puede"- expresa el reconocimiento de que las condiciones con las que recibió el país el actual Gobierno no fueron las mejores y que el escenario mundial y nacional no ha sido el más bonancible para la gestión de Funes.

Sin embargo, la población parece estar consciente de que las ayudas que recibe no resuelven sus problemas de una manera definitiva. La gente las valora, pero sigue pensando que la situación económica está difícil. La población sabe que la pobreza no se ha reducido $(47.8 \%$ ), está consciente de que la principal herramienta para salir de la pobreza —el empleo- no se está generando $(61 \%)$ y cerca de la mitad de la población (49.6\%) considera que la entrega de dinero a las familias pobres ayudará poco o nada a reducir la pobreza. En definitiva, la gente cree que la situación económica ha empeorado $(45.8 \%)$ con el presente Gobierno.

De acuerdo a lo que nos enseña la encuesta del IUDOP, la población percibe cambios que valora como positivos, pero pareciera decirnos que está consciente de que esos cambios no van a transformar la situación de su familia de manera duradera. Es como cuando se le da un analgésico a una persona que está sufriendo un dolor por alguna infección. Lo recibe agradecida porque le calma su padecimiento, aunque sepa que, una vez pase el efecto, el dolor puede volver.

\section{Seguridad pública: salidas de efecto inmediato o soluciones duraderas}

El problema más sentido por la población salvadoreña es la inseguridad y la violencia. A diferencia de lo que pasa con la situación económica, con la inseguridad la población no percibe revulsivos o analgésicos efectivos que alivianen esta pesada carga. Tan es así que no hay reparo en señalar el combate a la delincuencia como el principal fracaso del Gobierno (26\%), como una de las promesas menos cumplidas (51.9 \%) y como una de las dos principales tareas en la que se debería enfocar el Gobierno en su último año de gestión (37.5\%). 


\section{Dos visiones de la seguridad pública}

En cuatro años de gobierno, hemos visto a tres ministros de Justicia y Seguridad y tantos en la titularidad de la PNC. Esto - que en sí mismo ya habla de la inestabilidad en esta materia- preocupa sobre todo porque hemos visto dos proyectos o, mejor dicho, dos estilos distintos de enfocar la seguridad pública. Quizá es prematuro decir si los nuevos titulares (los terceros) continuarán el camino comenzado por sus inmediatos predecesores o le darán otro un rumbo diferente al enfoque de seguridad pública.

Las investigaciones contra exjefes de policía, algunos de los cuales fueron vinculados con el crimen organizado, fueron descalificadas por los legisladores por considerarlas persecución de carácter político partidario, pues la mayoría de los investigados provenían de las filas del Ejército
En esta materia, el Gobierno del presidente Funes comenzó su gestión con signos alentadores en el año 2009. El entonces nuevo gabinete de seguridad pública, presidido por Manuel Melgar en la cartera de Justicia y Seguridad y Carlos Asencio en la Dirección de la Policía Nacional Civil (PNC), hizo evidente su intención de recuperar la concepción original y constitucional de la PNC, una de las instituciones más importantes que parieron los Acuerdos de Paz de 1992. Hubo cambios en casi todas las estructuras de la PNC y se fortaleció la labor de la Inspectoría que investigó a altos jefes de la institución por nexos con el crimen organizado. Para octubre de 2009, a cuatro meses de iniciado el gobierno, de 216 policías que estaban siendo investigados por su presunta participación en delitos, 23 eran oficiales de alta graduación, 14 comisionados y 9 subcomisionados ${ }^{3}$.

Estos cambios fueron interpretados por la derecha como una cooptación de la institución por parte del FMLN, y argumentaban esta acusación señalando que los nuevos titulares de seguridad y los relevos en las jefaturas provenían de las filas de la exguerrilla. La labor de la Inspectoría fue boicoteada por la Asamblea Legislativa (Arena, GANA, PDC y PCN) que, para septiembre de 2010, había formado una comisión ad hoc para investigar las "extralimitaciones" de la funcionaria. Las investigaciones contra exjefes de policía, algunos de los cuales fueron vinculados con el crimen organizado, fueron descalificadas por los legisladores por considerarlas persecución de carácter político partidario, pues la mayoría de los investigados provenían de las filas del Ejército.

Pero los cambios no solo estuvieron en las personas que encabezaron las instituciones, sino en la actividad en contra de la delincuencia. Se comenzó a perseguir el crimen organizado proporcionándole algunos golpes, siendo el

3. Entre los altos oficiales que estaban siendo investigados, estaban el exdirector de la PNC, Ricardo Meneses, y el exsubdirector, Pedro González, vinculados por testigos de la Fiscalía con el narcotraficante José Natividad Luna Pereira (Chepe Luna). Douglas Omar García Funes, por omisión en la investigación y captura de Chepe Luna y facilitación de medios para la ejecución de ilícitos relativos al narcotráfico. Subcomisionado Godofredo Mirando, por peculado y por su participación en el caso de Los Perrones. 
más certero la desarticulación, en diciembre de 2010, de la banda conocida como "Los Perrones". Este grupo poderoso de empresarios que hizo su fortuna con el contrabando, lavado y narcotráfico, operaba la mayor empresa de transporte de droga de toda la región, que tenía su base en el oriente del país y que venían operando desde hace años con relativa normalidad en complicidad con bandas de la $\mathrm{PNC}^{4}$.

Pero este nuevo accionar de la policía coincidió con el inusitado aumento del número de homicidios en el país. Ya para diciembre de 2009, los homicidios presentaban una tasa de 71 por cada 100000 habitantes, muy superior a los 52 con que cerró el año precedente. No solo el número de homicidios creció, sino que cambió la modalidad de los asesinatos. Los cadáveres comenzaron a presentar signos de tortura, se multiplicaron las masacres que en no pocos casos incluyeron a niños y niñas, algunos recién nacidos. Cadáveres desmembrados comenzaron a aparecer en bolsas en las calles, cerca de estaciones de la policía. La barbarie llegó al colmo con el incendio de un bus con sus pasajeros adentro en la zona de Mejicanos el 19 de junio de 2010. Once personas murieron calcinadas, trece resultaron quemadas, entre ellos tres niños. Esta suele ser la típica reacción de los cárteles de la droga cuando se sienten acosados por las autoridades, envían el mensaje de lo que pueden hacer si se siguen tocando sus negocios. Esas escenas recordaban imágenes de México o de Honduras o la Colombia de hace unos años.

Y entonces comenzó la crítica pública. Los medios de comunicación que llenaban sus páginas y espacios con la violencia que azotaba al país, haciendo aparecer al Gobierno como ineficiente, cuestionaron la eficacia de la policía y comenzaron a pedir sus cabezas. Era lo que quería el crimen organizado. Al titular de la policía — comisionado Asencio-, se le criticó por muchas cosas, pero nunca nadie lo acusó de deshonesto, de corrupto. Los argumentos para destituirlo iban por otro lado y, en realidad, no eran lo importante.

En noviembre de 2011, el presidente Funes cedió y entonces comenzó el otro estilo de implementar la seguridad pública. El presidente, preocupado por mejorar la imagen y la eficacia de su Gobierno, decidió apostar por los militares para estar al frente de la seguridad pública, apuntándose otro mayúsculo error de su administración. Contraviniendo la Constitución y los Acuerdos de Paz, el camino recorrido hacia la "civilidad" de la PNC comenzó a desandarse en muy poco tiempo y se constituyó en uno de los mayores daños que este Gobierno heredará al país.

El error de origen de este estilo de la seguridad pública fue su diagnóstico sobre los responsables de la violencia y la inseguridad. El nuevo ministro de Justicia, incluso desde antes de ser nombrado como tal, comenzó a repetir que el mayor problema del país —en materia de seguridad - eran las pan-

4 De acuerdo al informe "Crimen organizado en Centroamérica: el triángulo norte", del Centro Internacional Woodrow Wilson, Los Perrones tienen amplios nexos con el cártel de Sinaloa y han buscado convertirse por sí mismos en una réplica de cárteles mexicanos, con nexos con Ecuador y Colombia. 
dillas juveniles y comenzó a anunciar la reducción de los homicidios en poco tiempo. Munguía Payés repetía que las pandillas eran las responsables del $90 \%$ de los homicidios, contradiciendo los mismos informes de la PNC que, en el 2011, les adjudicaban la autoría de solo el $26.25 \%$. Payés nunca demostró con pruebas lo que decía, pero nunca se ha cansado de repetirlo.

No es que las pandillas no sean un actor protagónico en la violencia del país. Es que no lo fueron nunca en las proporciones que declaraba el nuevo ministro de Justicia y Seguridad. Además, también hay que decir que son utilizadas por fuerzas oscuras del crimen organizado, como quedó demostrado en el incendio del microbús en Mejicanos o como lo constata el informe "Crimen organizado en Centroamérica: el triángulo norte", dado a conocer en el 2011 por el Centro Internacional Woodrow Wilson, al revelar que los pandilleros eran utilizados para dar seguridad y para labores de sicariato por parte de bandas como "Los Perrones".

Con el general Munguía Payés al frente del Ministerio de Justicia y Seguridad y el general Francisco Salinas al frente de la PNC, a la seguridad pública se le dio un enfoque militarista. Se cambiaron los mandos, las oficinas de inteligencia pasaron a ser controladas por militares, renunciaron el viceministro de la cartera y la titular de la Inspectoría de la PNC que estaba investigando a los altos oficiales por nexos con el crimen organizado. Algunos de los oficiales investigados regresaron a puestos claves de la PNC. El primer acto relevante (y probablemente el único hasta la fecha) del nuevo inspector de la PNC, Carlos Linares, fue archivar definitivamente, en septiembre de 2012, los expedientes contra el exdirector, el exsubdirector y otros seis altos oficiales de la PNC, por considerar que la investigación fue subjetiva y carente de elementos probatorios ${ }^{5}$.

A estas alturas, la tregua entre pandillas tenía seis meses de ser conocida en el país, desde que se hizo pública el 9 de marzo de 2012.

Y entonces los homicidios comenzaron a disminuir admirablemente rápido. De entre 14 y 15 al día hasta entre 5 y 6 diarios, una disminución que hasta antes de la tregua hubiera sido impensable. Y el discurso de Munguía Payés cobró sentido. Coincidentemente con las fechas del pacto entre las pandillas, comenzaron a disminuir los homicidios como lo había vaticinado el general. También esta disminución se produjo en coincidencia con el regreso de los altos oficiales a puestos claves de la PNC. Munguía Payés ahora defendía con números su tesis de que el $90 \%$ de los homicidios era responsabilidad de las pandillas y se granjeaba adulaciones al demostrar la inefectividad de las autoridades que le precedieron y la efectividad de los exmilitares procesados.

5. El nuevo inspector general de la PNC encontró fallas en el proceso de investigación interna contra el exdirector Menesses y los oficiales Óscar Antonio Aguilar Hernández, Godofredo Adalberto Miranda, Pedro Baltazar González Rodríguez, Douglas Omar García Funes, Luis Ernesto Núñez Cárcamo, Milton Oswaldo Escalón Fuentes y Eduardo Florencio Azucena López. 
Sin negar el innegable bien que representa la disminución de los homicidios sobre todo para la gente pobre que más sufre la violencia, nadie - ni Munguía Payés ni los que defienden el proceso con pandilleros- ha podido demostrar que la disminución de los asesinatos se deba a la tregua. Además, después de lo dicho antes, también se tendría derecho a concluir que esta disminución se debe a un pacto con los grandes del crimen organizado que dejaron de ser perseguidos y que hasta han dejado de ser mencionados como parte del problema de la violencia en el país. La utilización que el crimen organizado hace de las pandillas reforzaría también esta interpretación.

Durante los primeros dos años del Gobierno de Mauricio Funes, que coincidieron con la primera tanda de titulares al frente de la seguridad pública, la aceptación de la gestión gubernamental por parte de la población comenzó a decaer. En las encuestas del IUDOP, el Gobierno fue calificado con notas de 7.16 en agosto de 2009, 6.78 en mayo de 2010, 6.69 para noviembre del mismo año y 6.16 para mayo de 2011, cuando recién había comenzado la tregua entre las pandillas. Con el nombramiento de los militares al frente del Ministerio de Justicia y Seguridad y de la PNC, la percepción de la población comenzó a favorecer al Gobierno. En noviembre de 2011, se le adjudicó una nota de 6.47, en mayo de 2012 fue de 6.50 y, en noviembre del mismo año, de 6.74. En la más reciente encuesta del IUDOP de mayo de 2013, el Gobierno fue calificado con 6.87. Podríamos concluir que los cambios efectuados por el presidente le dieron los resultados esperados en lo referente a su imagen, pero, por lo que hemos dicho antes y por lo que diremos ahora, esto no es tan evidente.

\section{Una tregua sin validación social}

Comencemos diciendo que, a pesar de los esfuerzos del Gobierno por demostrar la efectividad de la tregua y de repetir que a la par de ella se persigue a los delincuentes, la opinión de la población es que, en este campo, el Gobierno no se ha desempeñado bien. El Gobierno ha revelado que, junto a la tregua, la PNC ha desarticulado más de 300 clicas y bandas del crimen organizado y culpa al órgano judicial de no procesar a los miles de delincuentes que la policía captura. En palabras del presidente Funes, solo en un año se efectuaron 83000 capturas, pero al final únicamente un $3 \%$ de los casos terminan judicializándose.

Pese a lo anterior, el combate a la delincuencia es considerado por la población como el principal fracaso del Gobierno (19.8\%): el $41.3 \%$ considera que la delincuencia ha aumentado con la actual administración, el $51.9 \%$ piensa que combatir la delincuencia es la segunda promesa menos cumplida (después de la creación de la fábrica de empleos, $61 \%$ ) y el $73 \%$ cree que la tregua ha ayudado a reducir poco o nada la delincuencia.

Si algo ha acompañado a la tregua desde su nacimiento, o por lo menos desde que se hizo pública, es la desconfianza generalizada por parte de la población y de diversas instancias de la sociedad civil. La reciente encuesta del IUDOP revela que el 83.1 \% de la población dice tener poca o ninguna 
confianza en la tregua entre las pandillas. Esta desconfianza tiene su correlato en que también, a lo largo de su vigencia, la tregua ha estado acompañada de la poca transparencia y de versiones diversas y hasta contradictorias con respecto a su contenido. Después de más de un año, la madeja se ha ido desenredando, pero no lo suficiente como para tener claridad de los objetivos, los participantes y los compromisos adquiridos por el Gobierno. Sin negar el bien que significa la reducción de homicidios, lo que más resiente la población es que, mientras son públicos los privilegios que se conceden a los líderes de las pandillas purgando penas, las extor-

Queda claro que la seguridad es una materia pendiente del Gobierno, como lo ha sido de todos los Gobiernos después de la guerra, porque es un problema estructural que no ha recibido respuestas estructurales siones, los robos y otros delitos siguen azotando a la familia salvadoreña. En la encuesta de mayo, 24 de cada 100 personas manifestaron haber sido víctimas directas de la delincuencia en el último año, un número mayor a los años precedentes.

Pero no solo la población ha manifestado su reserva frente al esfuerzo más publicitado por el Gobierno, también otros sectores como la Conferencia Episcopal, que emitió un pronunciamiento el 12 de mayo cuestionando los frutos de la tregua y su credibilidad. La guinda del pastel a esta desconfianza la puso la Sala de lo Constitucional de la Corte Suprema de Justica al declarar, el pasado 17 de mayo, inconstitucional el nombramiento de los militares al frente de la seguridad pública. Debido al ambiente de desconfianza alrededor de la tregua, la sentencia de la Sala ha tenido repercusiones más por la suerte que pueda correr la tregua que por la destitución de los militares.

El presidente Funes acató de inmediato la sentencia, aunque fue incapaz de detener algunos cuestionamientos hacia la Sala. Los nuevos titulares en el Ministerio de Justicia y Seguridad, Ricardo Perdomo y Rigoberto Pleités en la PNC, son los encargados de terminar un recorrido accidentado del Gobierno en esta materia. Para algunos analistas, quien seguirá llevando los hilos de la seguridad es David Munguía Payés, el cual, como el poder detrás del trono, ha sido designado asesor de la presidencia en materia de seguridad, sin especificar cuáles serán sus funciones. Pero para otros, los cambios efectuados por los nuevos titulares en la PNC —que incluyen la remoción de algunos de los oficiales cuestionados desde 2009 y las declaraciones anunciando el corte de privilegios para las maras- apuntan a que tomarán un camino distinto al de sus predecesores. Es prematuro emitir un juicio al respecto. Pero un año de gestión no da para impulsar otra concepción de la seguridad o para restablecer el proceso que se inició en el 2009 y que se truncó y se revirtió en el 2011.

Queda claro que la seguridad es una materia pendiente del Gobierno, como lo ha sido de todos los Gobiernos después de la guerra, porque es un problema estructural que no ha recibido respuestas estructurales. También queda bastante claro que la buena calificación del Gobierno de Mauricio Funes no se debe, por lo menos primariamente, a su desempeño en esta materia. Más bien se debe, como se apuntó arriba, a la implementación de los 
programas sociales que benefician a los más pobres, sobre todo por lo que tienen de soporte de una situación económica agobiante.

\section{Economía: un problema postergado}

El otro gran problema más sentido por la población tiene que ver con la situación económica. El 61 \% de los encuestados considera que el Gobierno no está cumpliendo su promesa de generar nuevos empleos y el $47.8 \%$ piensa que la promesa de reducir la pobreza no ha sido cumplida. Al preguntar a las personas por lo que consideran el principal fracaso del Gobierno, los problemas relacionados con la situación económica representan el $26.5 \%$ de las opiniones. En definitiva, la población percibe que la economía ha empeorado (45.8 \%) con el presente Gobierno.

La economía, como la violencia e inseguridad, tiene connotaciones estructurales. Las percepciones de la población sobre la situación económica tienen su correlato en la realidad del país. Es decir, al ser percepciones fundadas en la situación particular de la familia salvadoreña, se corresponden con la situación económica nacional.

Más de veinte años después de terminada la guerra, algunas voces argumentan que el conflicto armado fue por causas estrictamente políticas, en concreto, por la ausencia de las libertades democráticas. Escasa memoria tienen los que sostienen eso, pues, sin negar esa causa, la situación de exclusión y pobreza de una gran parte del pueblo salvadoreño fue la otra razón que provocó el conflicto armado. Los Acuerdos de Paz de 1992 tocaron frontalmente la causa política de la guerra, pero dejaron casi intacta la causa socioeconómica. A una década de la suscripción de los Acuerdos de $\mathrm{Paz}$, el secretario general de Naciones Unidas mencionó que, para el futuro del país, una de las principales tareas que se debía superar era "la exclusión económica y social generalizada" 6 . Pero pasaron los años y el modelo económico no solo no se transformó, sino que fue más fuertemente impulsado por los Gobiernos de turno.

Esta permanente postergación de enfrentar los problemas estructurales de la economía ha llevado a una situación que pone en peligro la viabilidad del país. La devoción al modelo neoliberal profesada por los Gobiernos que han venido después de la guerra ha empeorado la situación del país en general, aunque ha enriquecido exorbitantemente a una minúsculo grupos de actores económicos. El subempleo y desempleo afecta a prácticamente la mitad de la población económicamente activa. Cada año, de los cerca de 80000 nuevos bachilleres, 30000 buscan un trabajo donde ubicarse, y en la misma situación está parte de los 20000 nuevos profesionales que cada año se gradúan de las universidades nacionales. Sin embargo, de acuerdo a cifras oficiales, en los últimos tres años se han creado en promedio 25000 nuevos

6. Naciones Unidas. La situación en Centroamérica: procedimientos para establecer la paz firme y duradera, y progresos para la configuración de una región de paz, libertad, democracia y desarrollo, párrafo 14. 
empleos. Lo peor de esta situación es que no se ven perspectivas que enfrenten este problema de una manera seria y sostenida.

La deuda del Estado ronda los 13000 millones de dólares, sobrepasando el 55 \% del PIB, lo que, a juicio de las instituciones financieras internacionales, ha encendido las alarmas de la viabilidad económica del país. Desde hace muchos años, El Salvador es el país que menos crece en la región

\section{Pobreza y desigualdad}

Hay coincidencia en los análisis y las encuestas en situar a la precaria situación económica como uno de los dos grandes problemas del país. Sin embargo, a la hora de buscar las razones de la difícil situación, las opiniones son diversas.

El Salvador sigue siendo un país pobre porque nunca hay el suficiente tiempo para discutir cómo se va a reducir la pobreza; mientras tanto, los salvadoreños siguen arrodillados ante la pobreza cuando el tema de la pobreza es algo que puede resolverse.

(Ana Vilma de Escobar)

Hemos llegado al tiempo en que hablar de la pobreza como un problema estructural del país ha dejado de ser potestad de la gente de izquierda. La cita anterior, de la diputada de Arena Ana Vilma de Escobar, aparece en unas declaraciones que dio el 27 de febrero de este año. Admitir que la pobreza es lo que está en la base de la problemática económica y social del país ha dejado de ser problema incluso para los sectores que antes tenían proscrita esta palabra. Esto es un paso hacia delante que se debe reconocer. Sin embargo, dar un paso más y hablar de desigualdad es algo que todavía no está permitido en el lenguaje de los defensores del modelo económico. Y es que hablar de pobreza y de desigualdad no es lo mismo, sobre todo porque las consecuencias de tipificar el problema de una o de otra manera son muy diferentes. Si la pobreza es el problema medular, la correspondiente solución es la generación de riqueza. Esa es la conclusión a la que ha llegado la gran empresa privada y, por supuesto, nada mejor que su iniciativa para crear riqueza y bienestar. Lo que hay que hacer es impulsar la inversión privada, porque eso genera más empleos y más empleos reducen la pobreza. Así de sencillo. "La empresa privada es un factor importante para el desarrollo, generación de empleos, riqueza y reducción de la pobreza, por lo que con su labor lo único que hacen es prosperar el país y generar mejores condiciones de vida para los salvadoreños", afirmó Norman Quijano, candidato presidencial de Arena, en el Encuentro Nacional de la Empresa Privada, ENADE, en abril de este año.

Sin embargo, ubicar el problema económico en la desigualdad existente en el país remite a otro tipo de soluciones que incluyen el crecimiento económico, pero que exigen redistribuir el ingreso y la riqueza generada por toda la población. Esto es inadmisible para la gran empresa, porque hablar de desigualdad cuestiona medularmente el modelo a través del cual los grupos económicos se han enriquecido y los pobres se mantienen estancados. 
A los veinte años de los Acuerdos de Paz, nuevamente el secretario general de la ONU recodaba: "La atención a la desigualdad socioeconómica y los avances en la reforma de las instituciones de justicia frente a la inseguridad ciudadana son algunos de los retos que aún persisten a nivel nacional y regional"7.

La lucha contra la pobreza es una tarea que hay que continuar, pero es la desigualdad la que agrava el problema y lo convierte en el principal reto que el país debe enfrentar. El Salvador se ubica en la región más desigual del mundo en términos de ingreso económico, y el poderío de los grupos salvadoreños ha crecido a niveles desconocidos en los últimos años, aun en el periodo de crisis que todavía estamos viviendo. La desigualdad en el país tiene una particularidad con respecto al resto de países de América Latina. Al ser pequeño en territorio y densamente poblado, la pobreza convive con demasiada frecuencia al lado de una riqueza ostentosa y ofensiva.

Los niveles de la desigualdad económica se propagan hacia otros ámbitos de la vida provocando también otro tipo de desigualdades: en el campo educativo, en los servicios de salud, en el acceso a la vivienda, en la disponibilidad de los servicios básicos. Estudios del PNUD han demostrado que los salarios en el país son cada vez más desiguales al haber perdido su capacidad adquisitiva. Es decir, la desigualdad no se remite exclusivamente al ámbito económico, aunque esta sea la ocasión para generarla en otras dimensiones de la vida. El Banco Interamericano de Desarrollo ha dicho recientemente que la desigualdad es en América Latina el mayor freno al desarrollo sostenible. Además, con desigualdad, la cohesión social se hace muy difícil y, sin ella, también es tarea muy difícil pensar en el éxito de proyectos de alcance nacional.

\section{A manera de conclusión}

Como ya se evidenció, no es sencillo hacer una valoración de los cuatro años de gestión del actual Gobierno. No hay duda de que ha hecho cosas buenas y novedosas. Cerrar los ojos a la inversión pública en programas sociales es no hacer justicia a la realidad y, sobre todo, a lo que estos significan para los más pobres del país.

Aunque nuestro análisis se limita a los dos campos que más preocupan a la población, hay que reconocer también avances en otros campos que, de continuarse, pueden propiciar cambios más permanentes. Tal es el caso de lo hecho hasta hoy en materia de prevención frente al cambio climático, el establecimiento de una política energética más ecológica, los pasos en materia de transparencia, el aumento de la carga fiscal, aunque no deje de ser exigua, y el diseño en ciernes de un método de medición de la pobreza de una manera multidimensional. La aprobación de la Ley de Medicamentos es un paso importante, pero insuficiente en el entramado más amplio del derecho a la salud de la población.

7. Mensaje del Secretario General de la ONU, pronunciado por el Sr. Anders Kompass, director de la División de Operaciones de Terreno y de Cooperación Técnica, Oficina del Alto Comisionado para los Derechos Humano. 16 de enero de 2012. 
También la actual administración ha implementado cambios simbólicos sin que necesariamente cambien dinámicas de fondo. El reconocimiento y la celebración gubernamental de monseñor Romero, los actos de reconoci-

La inversión social en los últimos años ha crecido, pero en la transformación de las desigualdades a nivel estructural casi no se ha avanzado miento oficial de las masacres de los jesuitas y de $\mathrm{El}$ Mozote, pedir perdón por el papel del Estado durante la guerra son cambios de peso en el imaginario colectivo, sin que ello signifique que se cambien las dinámicas que estructuran la realidad del país. Igualmente, los frecuentes capítulos de enfrentamientos verbales entre la gran empresa privada -acostumbrada desde siempre a que su palabra sea atendida con prontitud- y el presidente de la República y su gabinete económico han suscitado, en parte de la población, la percepción de que el presidente no gobierna para los ricos empresarios que, dicho sea de paso, son uno de los sectores con menor credibilidad para la población. En realidad, esta percepción no ha tenido tampoco el correlato de cambios estructurales a través de la puesta en marcha de políticas económicas que toquen las bases del modelo de acumulación de los grupos económicos más poderosos del país.

Sin embargo, hay que decir que el Gobierno no ha resuelto ni comenzado a resolver serias injusticias estructurales de larga data en El Salvador. Nuestro balance quiere captar la complacencia de la gente frente a los programas sociales, pero también el desencanto ante la esperanza de cambios más profundos que no han llegado a cristalizarse.

La inversión social en los últimos años ha crecido, pero en la transformación de las desigualdades a nivel estructural casi no se ha avanzado. Los programas sociales que benefician a los sectores más pobres se están encaminando a transformarse en programas clientelistas, sirviendo, como en otros tiempos, más de materia prima para las campañas electorales que para la transformación duradera de la situación de las familias más pobres. La encuesta del IUDOP refleja que los mayores apoyos del Gobierno y del partido gobernante están en los sectores que antes fueron, precisamente, la base social de Arena mientras estuvo gobernando. Por contrapartida, los otrora apoyos del FMLN — sectores profesionales, medios, urbanos- le han retirado su simpatía en los últimos años.

Los programas sociales, con el innegable bien que representan para las familias más pobres, carecen del factor de sostenibilidad tan necesario para sentar las bases de un desarrollo humano y más equitativo. Como no son fiscalmente sostenibles en el tiempo, el recurso al endeudamiento ha llegado prácticamente al límite porque al Gobierno le faltó voluntad política -apoyo popular lo tuvo desde el principio- para aplicar una reforma fiscal que tocara a los sectores económicos que históricamente se han enriquecido sin pagar lo que les corresponde. Los grandes empresarios del país se conciben a sí mismos como los generadores de riqueza, de empleos y como los abanderados del desarrollo. Ellos deben recordar, como ha dicho el BID, los muchos riesgos que se corren mientras persista y aumente la desigualdad. Esa que hace que nuestro país esté en el índice de desarrollo humano cerca 
de 20 puntos por debajo de la posición que ocuparía si la desigualdad se redujera. El PNUD estimó que, para el período 2007-2008, el 80 \% de nuestra población carecía de un ingreso decente. Seguramente, la mayoría de estos empleos son generados por la gran empresa que se ufana de crear el $80 \%$ de los empleos formales. La gran empresa privada salvadoreña es tan voraz que no está dispuesta a reducir los márgenes de ganancia y renunciar a privilegios egoístas que afectan a la población. Si los empresarios se dieran cuenta de que su mejor negocio estaría en la mejora de la capacidad adquisitiva y de consumo de los trabajadores, entonces accederían a mejorar la situación de la gente, como dijo Cabral, aunque sea por negocio.

En materia de seguridad, mientras se entienda la problemática de la violencia $e$ inseguridad vinculándola casi exclusivamente a las pandillas y se borre del escenario al crimen organizado que tiene una importante cuota en el ejercicio de la violencia, incluso en la provocada por las pandillas, los pasos que se den no serán los adecuados ni los suficientes. Mientras no se aborde la problemática de la violencia desde un análisis histórico de la impunidad, no van a tocarse los dinamismos de fondo que la sustentan. La renuencia del Estado salvadoreño para investigar, capturar, procesar y juzgar a los responsables de graves violaciones de los derechos humanos durante y después del conflicto armado sienta un "precedente negativo en cuanto al respeto de los derechos humanos, posición oficial que se mantiene desde el momento de la publicación y la divulgación del Informe de la Comisión de la Verdad"8.

En realidad, los dos problemas más sentidos por la población —violencia y crisis económica - deben abordarse desde un tronco común, pues ambas son manifestaciones de problemas estructurales. Para expresarlo, nos remitimos a lo que dice el pronunciamiento de la UCA sobre la situación de violencia e inseguridad, del 4 de junio pasado: "La raíz de la violencia en sus diversas manifestaciones está en la desigualdad económica y social producida por un sistema estructuralmente violento, que empuja a su gente fuera del país o hacia las sendas torcidas del crimen, y que se resiste a reformas de equidad básica, incluso en el sistema tributario. Un sistema que considera como un dios al dinero y cuya dinámica hace crecer desmesuradamente los beneficios de una pequeña minoría, mientras disminuyen dramáticamente las posibilidades de bienestar de la mayoría".

8. Informe del Comisionado Nacional para los Derechos Humanos, a los 20 años de la firma de los Acuerdos de Paz, pág. 31. 